\title{
O DESIGN DE PRÓTESES OPEN SOURCE PARA MEMBROS SUPERIORES POR MEIO DA ANÁLISE DOCUMENTAL DE PROJETOS
}

\section{THE DESIGN OF OPEN SOURCE PROSTHESIS FOR HIGHER MEMBERS THROUGH THE DOCUMENTARY ANALYSIS OF PROJECTS}

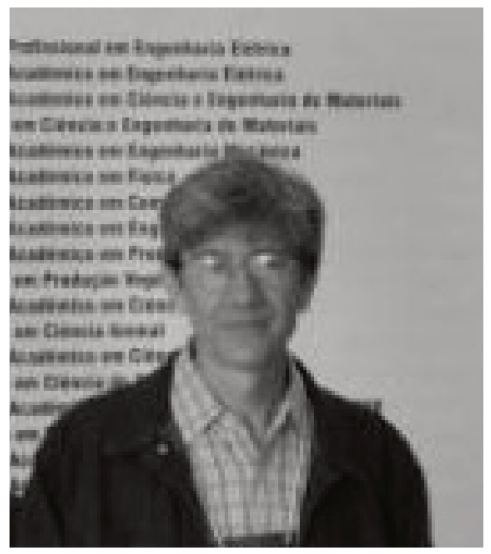

\author{
Milton José Cinelli \\ Doutor em Chimie Physique Option Matériaux - Université \\ de Limoges - França \\ Professor Permanente do Programa de Pós-Graduação \\ em Design da Universidade do Estado de Santa Catarina \\ (UDESC) \\ milton.cinelli@udesc.br
}

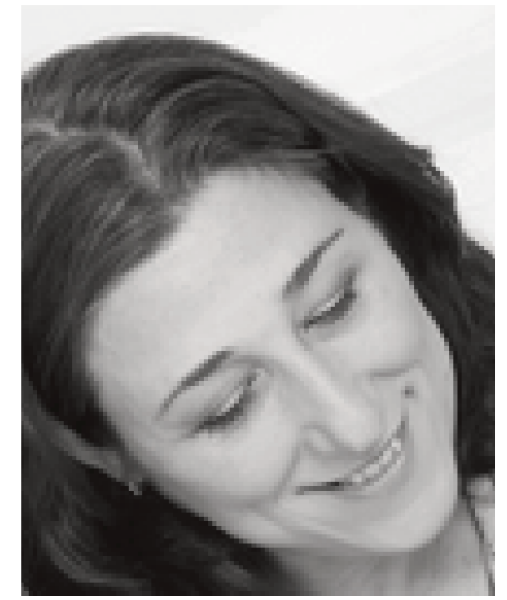

\section{Susana Cristina Domenech}

Doutora em Engenharia de Materiais pela Universidade Federal de Santa Catarina

Professora associada no Centro de Ciências da Saúde e do Esporte

scdomenech@gmail.com 


\section{LOGO}

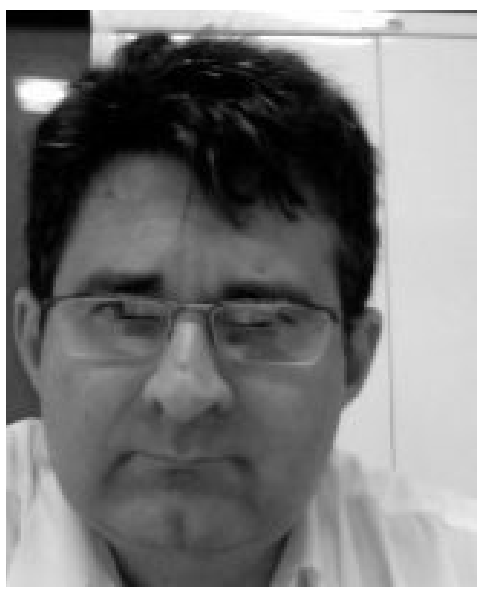

\section{Marcelo Gitirana Gomes Ferreira}

Doutor em Engenharia mecânica pela Universidade do Estado de Santa Catarina (UFSC)

Professor associado no departamento de Design da Universidade do Estado de Santa Catarina (UDESC) marcelo.gitirana@gmail.com

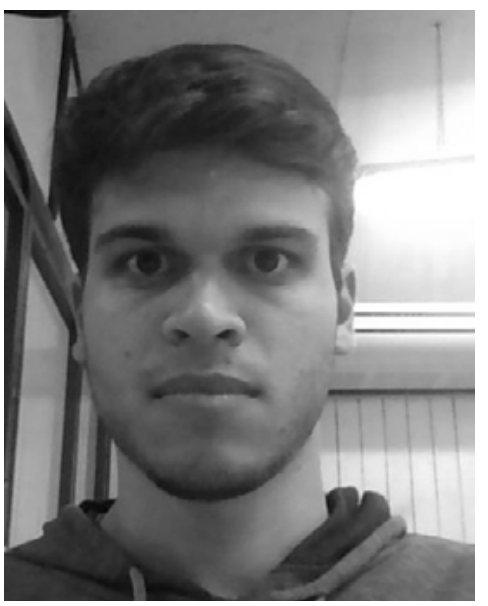

\section{Ricardo Schwinn Rodrigues}

Mestrando no Programa de Pós Graduação em Design (PPGDesign) da Universidade do Estado de Santa

Catarina (UDESC)

rsr404@hotmail.com 


\section{RESUMO}

Indivíduos amputados de regiões pouco desenvolvidas e em desenvolvimento, possuem acesso limitado a dispositivos protéticos No bojo deste cenário, surgem grupos colaborativos para o desenvolvimento de próteses de baixo custo fabricadas por impressão 3D, com distribuição livre e gratuita (open source), visando o acesso e melhoria da qualidade de vida a populações menos favorecidas economicamente, em escala global. Nesta perspectiva, o objetivo do presente estudo foi analisar as características relacionadas aos fatores humanos e ergonômicos presentes em projetos de design de próteses open source para os membros superiores. $O$ estudo documental teve como amostra 09 projetos veiculados pelo site E-Nabling The Future. Os projetos analisados sugerem um ganho em relação à acessibilidade das próteses open source em comparação às próteses funcionais devido à livre distribuição, ao baixo custo de produção e manutenção e à customização antropométrica e estética desses dispositivos. Além disso, os fatores humanos e ergonômicos dos dispositivos, embora presentes nos projetos, são pouco consistentes, podendo implicar no uso e no engajamento do usuário com os dispositivos. No entanto, os projetos de próteses open source são projetos de design coerentes para os contextos e objetivos a que seu desenvolvimento se propõe, sendo capazes de atuar como alternativa acessível às próteses convencionais, possibilitando a realização de atividades da vida diária de uma parcela considerável da população.

\section{PALAVRAS CHAVE:}

Design de próteses, próteses para o membro superior, open source.

\section{ABSTRACT}

Amputees from underdeveloped and developing regions have limited access to prosthetic devices. In this scenario, collaborative groups arise for the development of low-cost prosthetics devices, made by 3D printing, with open source distribution, aiming the access and improvement of the life quality on economically disadvantaged populations, on a global scale. In this perspective, the objective of this study was to analyze the characteristics related to the human factors and ergonomics, present in the design of open source prostheses for the upper limbs. The documentary study had as subjects 9 projects conveyed by the E-Nabling The Future website. In addition, the human factors and ergonomics of the devices, although present in the designs, are lack in consistent and can imply in the user's use and engagement with the devices. However, the open source prostheses are coherent design projects considering the contexts and objectives their are intended, being able to act as an accessible alternative to conventional prostheses, enabling the accomplishment of daily life activities of a considerable part of the population. 


\section{KEY WORDS:}

Prosthetic design, upper limb prosthesis, open source.

\section{INTRODUÇÃO}

A amputação caracteriza-se pela retirada total ou parcial de um dos membros. No Brasil, as informações sobre o assunto são imprecisas. Dados do Ministério da Saúde (2014) relativos a 2011, estimam que as amputações de membros superiores correspondem a 15\%. Estas estão diretamente relacionadas com a perda ou limitações na capacidade funcional e laboral do indivíduo, resultante da perda da integridade da sua capacidade de manipulação. Durante o processo de reabilitação de pacientes amputados, o uso de próteses pode ser uma alternativa para a retomada das Atividades da Vida Diária (AVD).

As próteses são dispositivos médicos e, no caso dos membros superiores, têm como função principal a melhoria da capacidade de manipulação do indivíduo, contribuindo para a realização de AVD, a participação em atividades laborais, a ampliação da performance em determinada atividade, além de contribuir com a aceitação da aparência (HECKATHORNE et al, 2002). Sua projetação depende, entre outros fatores, das metas funcionais do indivíduo e da condição do membro residual, possibilitando a simulação das funções de movimento e melhoria de sua funcionalidade (MUSICUS; DAVIS 2013). Também podem ser consideradas Tecnologia Assistiva (TA). Cook e Polgar (2013) conceituam TA como qualquer "produto, instrumento, equipamento ou tecnologia adaptados ou especialmente projetado para melhorar o funcionamento de uma pessoa com deficiência", seguindo definição apresentada pela OMS (2001).

A funcionalidade das próteses impacta diretamente a capacidade funcional dos indivíduos, em vista disso, o projeto desses dispositivos deve valorizar, sobretudo, a usabilidade e a segurança do usuário. Quando vistos sob a ótica dos fatores humanos, faz-se necessário considerar os seguintes aspectos: o conhecimento das características dos usuários (paciente, enfermeiras, familiares), a expectativa do usuário quanto à funcionalidade e uso do equipamento, o ambiente (temperatura, iluminação, acessibilidade) e os aspectos culturais (acesso a treinamento e assistência) nos quais o dispositivo e usuário estão inseridos (MARTIN et al 2010). Portanto, uma relação satisfatória entre o design e dispositivos médicos está pautada na atenção aos aspectos ergonômicos do projeto.

Ao considerar os fatores humanos e a ergonomia, é indispensável levar em conta o acesso do indivíduo às próteses, que está limitado à sua capacidade financeira e às práticas do sistema de saúde de seu país. Nesse contexto, observa-se que, segundo a OMS $80 \%$ das pessoas com algum tipo de deficiência física vivem em países 
de baixa renda, que têm seu acesso à próteses restringido, devido aos altos custos, somado à condição sócio econômica (RESNILK, 2012). Esta realidade é agravada em crianças, pois, seu rápido desenvolvimento exige diferentes tamanhos de próteses a cada estágio de crescimento, tornando elevados os custos de aquisição e manutenção das mesmas (ZUNIGA et al, 2015).

O acesso a próteses funcionais e de baixo custo em países de baixa renda, é o eixo central da estratégia de grupos de desenvolvedores como E-Nabling the Future (EUA), OpenBionics (Reino Unido) e a Exii (Japão), que reduzem o custo de seus produtos através da utilização de técnicas digitais de produção e distribuição. As próteses desenvolvidas por esses grupos são open source (de livre distribuição uso e modificação) disponibilizadas gratuitamente em plataformas online de distribuição de arquivos digitais (projetados em softwares CAD - computer aided design). A produção de suas partes ocorre por impressão 3D e a montagem realizada pelo próprio usuário. Esse processo digital e colaborativo constitui uma nova forma de projetação denominada open design. Na linha desta dinâmica, os projetistas trabalham de forma autônoma, em colaboração, sendo eles próprios os responsáveis pelo desenvolvimento, produção e distribuição dos projetos. Contudo, essa nova forma de projeto, possui a desvantagem de gerar projetos de design inconsistentes em seus aspectos funcionais, estéticos e simbólicos, umas vez que o projeto não é, necessariamente, desenvolvido por profissionais graduados em design e áreas afins.

Considerados esses aspectos, o objetivo deste estudo é analisar as características projetuais dos das próteses open source disponibilizados pela comunidade colaborativa de desenvolvedores nomeada E-Nabling the Future, pelo viés dos fatores humanos e da ergonomia.

\section{PROJETOS DE PRODUTOS OPEN SOURCE, O OPEN HARDWARE E O OPEN DESIGN}

Projetos open source preveem a livre distribuição, utilização e eventual modificação de um produto ou de suas partes, desde que o autor original seja creditado (KATZ, 2011). Desse modo, o principal aspecto inerente aos projetos open é sua "abertura" (opennes). Para Avital (2011) a abertura de um projeto possui três esferas básicas: a visualização (view) modificação (modify) e uso (use). Visualizar diz respeito ao compartilhamento de algo e suas informações, modificar diz respeito ao compartilhamento dos resultados e as melhorias efetuadas em um projeto e usar refere-se ao compartilhamento da propriedade de algo. Projetos open source, em sua maioria, estão protegidos sob licenças Creative Commons (CC), garantindo o livre uso, modificação e a redistribuição de projetos. 
Outro conceito pertinente ao contexto é o de Open Hardware, que segundo Acosta (2009) "é o hardware (objeto físico) disponível gratuitamente sob uma das licenças de código aberto juridicamente reconhecidas" incluindo esquemas, diagramas e regras de design que podem ser usadas e modificadas e redistribuídas com pouca ou nenhuma restrição. O conceito difere-se ligeiramente do open software, já que um produto open hardware sempre terá o envolvimento da indústria tradicional, pois requer matéria-prima (física) disponível no mercado para a construção de componentes do projeto.

Por sua vez, o open design refere-se aos aspectos envolvidos na projetação open. Para Avital (2011) e Kadushin (2010) o open design concerne a livre distribuição de projetos e suas informações técnicas, em formato digital, possibilitando sua modificação para adequação aos requisitos de usuários específicos. A fabricação desse projeto é, preferencialmente, realizada pelo próprio usuário ou grupo de usuários, utilizando os meios de produção aos quais possui acesso, normalmente, ferramentas de prototipagem rápida como a impressão 3D. Nota-se, portanto, que o processo do open design depende intrinsecamente do usuário, pois é ele que consome, materializa, modifica e redistribui os projetos, tornando-se em algum momento do processo, o projetista/designer. Em linhas gerais, no viés do open design, o designer é capaz de realizar todo o processo de desenvolvimento de produto, da concepção à fabricação e venda.

Devido a sua natureza aberta, os produtos resultantes do open design são produtos open source, que com frequência utilizam-se de produtos open software e open hardware como componentes do projeto. Essa ótica open é uma dos componentes que Anderson (2012), define como "a nova revolução industrial", referindo-se ao uso de métodos alternativos de desenvolvimento, fabricação e distribuição de bens de consumo. Para o autor, as características básicas do movimento são: o uso de ferramentas digitais para o desenvolvimento e prototipagem de produtos, a cultura de compartilhamento e colaboração online e a adoção de formatos digitais em comum, facilitando o compartilhamento e fabricação. Em suma, essa nova revolução é ocasionada pela digitalização da informação e dos meios de produção e, particularmente, pelo grupo de indivíduos que produzem seus próprios objetos usáveis a partir do acesso à hardware e à software digitais. Esse grupo de indivíduos, somado a esse novo contexto digital, é denominado pelo autor como o Movimento Maker.

Para Anderson (2012), essa revolução não está totalmente estabelecida, mas já é considerada como realidade. Prova disso é a existência de um ecossistema específico que fornece todas as ferramentas para o desenvolvimento, produção e distribuição de conteúdo/produtos. No ecossistema Maker (NEVES, 2014) a infraestrutura é descentralizada e composta de locais físicos - Maker Space - que possuem as ferramentas 
(impressoras 3D, CNC, etc.) necessárias para desenvolvimento e fabricação de projetos, como os FabLab, uma rede global de laboratórios que visa a inovação colaborativa. Plataformas de compartilhamento como Instructables e Thingiverse e até mesmo serviços de produção como Ponoko, que produzem projetos enviados pelos desenvolvedores, fazem parte do processo.

Contudo, a natureza projetual aberta e livre pode apresentar aspectos negativos, pois, ao tornar o usuário um co-designer capaz de modificar projetos, o desenvolvimento open estará sempre limitado à capacidade do projetista em dominar, em algum nível, expertises como o design, eletrônica, softwares $C A D$, processos produtivos, entre outros, podendo resultar em produtos de baixa qualidade, funcionalidade e estética, ou plataformas já estabelecidas podem ser modificadas para produtos menos estáveis e serem amplamente consumidos e compartilhados (DE MUL, 2011); (ACOSTA, 2009).

\section{USO DA IMPRESSÃO 3D NO DESENVOLVIMENTO DE PRODUTOS}

Os projetos open source preconizam o uso de meios de fabricação alternativos, costumeiramente, ferramentas de prototipagem rápida, que apresenta como principal processo a impressão 3D. Essa última é uma tecnologia de manufatura aditiva, que produz objetos através da adição de camadas sobrepostas de material em seções horizontais, a partir dos dados de um modelo 3D criado em um software específico, podendo ser projetado em software ou escaneado com um scanner 3D (VALPREDA, 2015).

A impressão 3D distingue-se dos meios tradicionais de produção devido à sua versatilidade e capacidade em diminuir o ciclo de desenvolvimento e o custo do produto. Teoricamente, uma impressora 3D pode ser empregada como única ferramenta à fabricação, imprimindo produtos funcionais que já constam com partes móveis, engrenagens e elementos de fixação em uma única sessão de impressão. Ademais, é capaz de imprimir formas variadas, com diferentes graus de complexidade e dimensões (LIPSON \& KURMAN, 2013).

É também, uma alternativa viável para a personalização/customização de produtos em massa e em pequenas quantidades, impactando na redução de custos ao diminuir o processo logístico, não necessitando de moldes para a fabricação de peças, tão pouco, a necessidade de estoque, pois, os produtos podem ser produzidos por demanda. Outra característica é a possibilidade de reciclar em até 98\% do material desperdiçado, o que a torna ecologicamente correta (BERMAN, 2011). Já Conner et al (2014), observam que a impressão 3D possui uma clara vantagem produtiva quando utilizada na produção de produtos de alta complexidade de construção e alto grau de customização. Portanto, a possibilidade de produzir formas adaptadas à singularidade dimensional do corpo humano, somados à sua 
vantagem econômica, torna a impressão 3D uma excelente opção para a produção de produtos médicos como próteses e órteses (LIPSON \& KURMAN, 2013).

\section{TIPOS DE PRÓTESES PARA OS MEMBROS SUPERIORES E SEUS ELEMENTOS}

As próteses funcionam como ferramentas que auxiliam nas AVD do indivíduo amputado ou em atividades específicas, com particular atenção ao desempenho dos movimentos, pois, cada tarefa exige movimentação específica e o treinamento do indivíduo no uso da prótese (MUSICUS; DAVIS, 2013). O uso de próteses decorre da opção do indivíduo amputado em utilizá-las a partir da recomendação médica durante o processo de reabilitação. Esse processo deve considerar os aspectos da saúde, da capacidade funcional, social, cognitiva, cultural e financeira do indivíduo e deve ser resultado de um trabalho realizado por uma equipe multidisciplinar composta por profissionais e familiares, com a devida atenção dada às atividades laborais, às AVD e às atividades de lazer almejadas pelo indivíduo, potencializando sua independência. Além desses, outros fatores críticos para o engajamento do paciente na utilização de próteses são o treinamento quanto à utilização, manutenção e benefícios das mesmas, evitando assim, o abandono do dispositivo pelo indivíduo amputado (NGD, 2014).

Próteses para os membros superiores básicas são compostas de: I) Soquete: interface entre o membro residual e a prótese; II) Sistema de suspensão: que fixa a prótese no membro do indivíduo, durante o uso; III) Articulação: que movimenta a prótese no espaço; IV) Dispositivo terminal: que manipula objetos, pode ser similar a ganchos/ garras ou a mãos, normalmente, em uma sistema de preensão (movimento que a mão realiza em torno de um objeto para segurá-lo) em três pontos (simulando a movimento em pinça tridigital: quando o apenas os dedos polegar, indicador e médio envolvem o objeto). Os dispositivos terminais dividem-se em: I) Passivos: quando controladas pelo membro contralateral, ou II) Ativos: quando os mecanismos da prótese realizam o movimento de preensão (MUSICUS; DAVIS, 2013).

No que diz respeito à sua classificação, as próteses para os membros superiores são classificadas em cinco tipos, segundo Heckathorne (2002): I) Cosméticas: que simulam a fisiologia do membro, sem componente terminal ativo; II); Mecânicas (controladas pela energia do corpo): cabos ativam o componente terminal da prótese a partir do movimento do membro residual; III) Controlados por energia elétrica: controlada através da atividade mioelétrica do corpo; IV) Híbrida: que combina componentes controlados por energia elétrica e por energia do corpo; V) De Função especial: desempenham uma função específica (ex: para a prática específica de esportes). 


\section{PRÓTESES OPEN SOURCE PARA OS MEMBROS SUPERIORES}

Projetos de próteses open source para os membros superiores visam sanar a limitação ao acesso dos mesmos em países de baixa renda, nos quais a saúde é precária. A abordagem open garante custos mais baixos às próteses, pois os arquivos para a sua fabricação são digitais (desenvolvidos em software CAD) e fabricados em impressoras 3D, possibilitando alto grau de reprodutibilidade do dispositivo e de suas partes, o que facilita a distribuição e reduz os custos e o tempo de manutenção. Ainda, a possibilidade da adaptação das próteses a diferentes medidas antropométricas de membros amputados, tornam os dispositivos funcionais e práticos, com funcionalidades básicas como o abrir e fechar dos dedos (Slade et al 2015).

O primeiro projeto de prótese open source utilizando a impressão 3D surge em 2012 na África do Sul, com Richard van A, por meio de sua iniciativa chamada Robohand $O$ projeto é um projeto open hardware de prótese para o membro superior mecânica, controlada pelo movimento do pulso e distribuída digitalmente. Por se tratar de um sistema open hardware, é fabricada com partes impressas em impressão 3D, e partes adquiridas comercialmente como parafusos, fios tensionadores e placa termoplástica. O dispositivo custa cerca de US\$ 5,00 para as partes impressas e US\$2000,00 no total (Robohand, 2016a); (Robohand 2016b). Além da RoboHand existem outros grupos e organizações de desenvolvedores como a E-nabling the Future (EUA), voltada ao desenvolvimento de próteses mecânicas para crianças, a OpenBionics (Reino Unido) com a prótese AdaHand, e a Exii (Japão) com a prótese Hackberry, essas duas últimas ainda em fase de protótipo.

A E-Nabling The Future, foco deste estudo, é uma comunidade colaborativa global iniciada por Ivan Owen em 2013, nos EUA, composta por designers, professores, engenheiros e entusiastas, que objetivam o desenvolvimento e a distribuição de próteses para os membros superiores. São dispositivos open source, de baixo custo (variando entre US\$20 e US\$50 para a impressão de suas partes) voltados para crianças a partir dos 3 anos (E-nabling The Future 2016a, 2016b). Os desenvolvedores denominam suas próteses como próteses experimentais, ferramentas que auxiliam em funções simples como segurar garrafas, copos, andar de bicicleta, entre outros, que devem ser utilizadas com o acompanhamento de adultos ou profissionais da saúde. E, no momento, não devem ser encaradas como próteses funcionais, pelo fato de comportarem somente baixas cargas e a carência de testes biomecânicos consistentes. (E-nabling The Future 2016c).

As próteses são disponibilizadas digitalmente no site da inciativa http://enablingthefuture.org/, contendo os arquivos para impressão das partes do dispositivo e os manuais de montagem. O site da E-nabling The Future, contexto e análise do presente estudo, reúne 15 próteses para o membro superior. 


\section{SATISFAÇÃO DO USUÁRIO NO USO DE PRÓTESES PARA O MEMBRO SUPERIOR}

É crescente a valorização dos aspectos relativos à usabilidade e à segurança no desenvolvimento de dispositivos médicos e tecnologias assistivas. Aspectos esses, que se relacionam com a maneira com que o objeto interage com o usuário e, portanto, englobam os fatores humanos e ergonômicos desses dispositivos. Ou seja, é fundamental a atenção às necessidades do paciente, seu contexto social e cultural, às características do dispositivo e o seu uso pretendido. A desatenção a esses aspectos pode fazer com que o usuário não perceba a necessidade em utilizar a prótese.

Vasluian et al (2013), ao pesquisarem o uso de próteses em crianças e adolescentes, observaram que os motivos para o uso de próteses está relacionado a aspectos cosmético, funcional e físico. $\mathrm{O}$ aspecto cosmético é especialmente relevante para crianças e adolescentes, pois facilita a sua relação com o seu próprio corpo e com outras pessoas. $\mathrm{O}$ aspecto funcional, diz respeito à capacidade de manipulação e auxílio nas AVD, e o aspecto físico, relaciona-se com o sentimento de que as próteses podem ajudar no seu desenvolvimento muscular.

Por sua vez, Cordella et al (2016) apresentam requisitos para o desenvolvimento de próteses a partir das necessidades e anseios reportados pelos usuários, que são a necessidade da prótese em realizar AVD, oferecer feedback sensorial, realizar atividades com maior força e maior precisão (coordenação) na realização de tarefas, bem como, próteses com nível maior de antropomorfismo, capacidade de mudança na orientação e posição de objetos manipulados com a prótese, capacidade de movimentar cada dedo individualmente, assim como, a movimentação do punho e melhoria da durabilidade da prótese. Davidson (2002), ainda aborda a temperatura como um fator importante, pois o suor, em climas quentes, pode desestimular o uso da prótese.

\section{MÉTODO}

O estudo caracteriza-se como uma pesquisa documental, com análise de 09 projetos de próteses para membros superiores da comunidade de desenvolvedores de próteses E-nabling The Future, a partir de sua documentação e arquivos para a fabricação. As informações foram retirados através do acesso ao site http://enablingthefuture.org/, centrando-se apenas nas próteses movidas a energia mecânica ativada pelo pulso (wrist powered hand devices), disponíveis no link: http://enablingthefuture.org/wrist-powered/.

A análise ocorreu a partir da observação das imagens das próteses, da leitura das descrições dos projetos, dos manuais contendo instruções de montagem e do 
download dos arquivos das partes das próteses disponíveis a partir dos links apresentados no Quadro 01. Foram levados em consideração os aspectos relacionados aos fatores humanos no projeto de próteses, capazes de influenciar no uso dos dispositivos pelos usuários, os aspectos foram categorizados e denominados pelos autores deste estudo como: I) acessibilidade; II)estética ; III) fabricação e manutenção; IV) instruções treinamento e segurança; $\mathrm{V}$ ) conforto; $\mathrm{VI}$ ) aspectos funcionais. Os links foram acessados no período que compreende os meses de julho a agosto de 2016. Os dispositivos selecionados para a análise documental, bem como suas respectivas páginas nas quais estão disponibilizados, serão apresentados no Quadro 01, bem como, a imagem dos produtos identificados e analisados, conforme apresentado na Figura 01.

\begin{tabular}{ll}
\hline Prótese analisada & Página em que é disponibilizada \\
$\begin{array}{l}\text { Raptor Reloaded } \\
\text { Phoenix Hand }\end{array}$ & http://enablingthefuture.org/upper-limb-prosthetics/raptor-reloaded/ \\
Osprey Hand & http://enablingthefuture.org/phoenix-hand/ \\
Raptor Hand & http://enablingthefuture.org/osprey-hand/ \\
Talon Hand 3.0 & http://enablingthefuture.org/upper-limb-prosthetics/talon-hand/ \\
Cyborg Beast & http://enablingthefuture.org/upper-limb-prosthetics/cyborg-beast/ \\
Odysseus Hand & http://enablingthefuture.org/upper-limb-prosthetics/odysseus-hand/ \\
Flexy Hand 2 & http://enablingthefuture.org/upper-limb-prosthetics/the-flexy-hand/ \\
K1 Hand & http://enablingthefuture.org/k-1-hand/ \\
\hline
\end{tabular}

QUADRO 01: lista das próteses analisadas neste estudo e os respectivos links, a partir dos quais, os arquivos de fabricação são disponibilizados. 

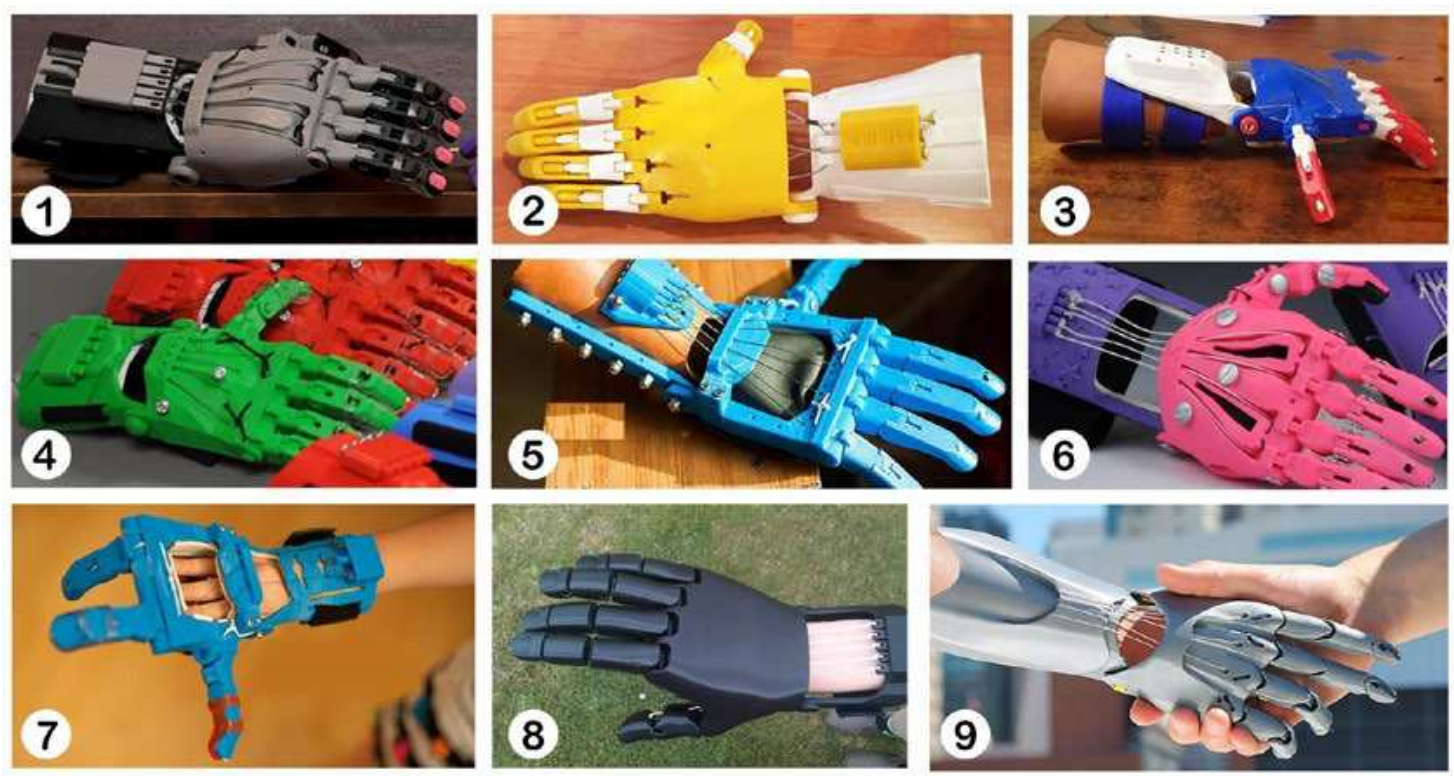

FIGURA 01. Imagens das Próteses analisadas 1: Raptor Reloaded. 2: Phoenix Hand. 3: Osprey Hand. 4: Raptor. 5: Talon Hand. 6: Cyborg Beast. 7: Odysseus Hand. 8: Flexy Hand 2. 9: K1 Hand. Imagens adaptadas de: 1:(E-nabling The future, 2016d); 2: (E-nabling The future 2016e); 3:(E-nabling The future, 2016f); 4:(E-nabling The future, 2016g); 5: (E-nabling The future, 2016h); 6: (E-nabling The future, 2016i); 7: (E-nabling The future, 2016j) 8: (E-nabling The future, 2016k); 9: (E-nabling The future, 2016l).

\section{RESULTADOS}

A seguir são apresentadas as informações sobre próteses movidas a energia mecânica ativada pelo pulso, obtidas mediante análise de projetos presentes no site da E-Nabling The Future.

\section{ACESSIBILIDADE}

Os projetos das próteses estão disponibilizados na plataforma online de compartilhamento de arquivos digitais Thigiverse (http://thigiverse.com), exceto os arquivos da K1 Hand que estão disponibilizados no site http://3dprint.nih.gov/. Os arquivos contêm uma ou mais partes da prótese, todos estão em formato .stl (STereoLithography). No que diz respeito aos componentes não impressos das próteses, as páginas de download dos arquivos da Raptor Hand e a Cyborg Beast, disponibilizam links para a aquisição de kits para a montagem das próteses, disponíveis em: http://shop3duniverse.com/collections/3d-printable-kits. No que diz respeito aos componentes não impressos das demais próteses, esses devem ser adquiridos comercialmente pelo usuário, portanto, não há como descrever neste estudo, o acesso 
do usuário a esses componentes, pois a disponibilidade e o preço de cada componente podem variar em cada país ou região na qual o usuário pretende adquiri-los.

\section{ESTÉTICA}

Na Tabela 1, é apresentado um comparativo entre a similaridade das próteses com a aparência e da mão Humana (aparência antropomórfica), com a aparência externa da mão humana, no que diz respeito à estética da prótese. A tabela está dividida em dois segmentos, sendo o 1, relativo a elementos da mão humana incorporados na prótese, positivo e o 2, relativo a elementos funcionais das expostos da prótese, negativo. A análise foi realizada observando imagens de cada projeto disponível no site E-nabling the future, a partir dos seguintes requisitos:

1. Elementos da mão humana incorporados na prótese: presença dos 05 dedos; presença de todas as articulações dos dedos; representação das unhas; presença da parte dorsal da mão e presença da palma da mão.

2. Elementos funcionais expostos da prótese: elementos de fixação (EF) expostos na parte dorsal da mão; EF expostos na parte palmar da mão; EF expostos no parte superior dos dedos; fio tensionador exposto na parte dorsal da mão e fio tensionador exposto no antebraço da prótese.

\begin{tabular}{lccccccccc}
\hline \multicolumn{10}{c}{ Número da Prótese } \\
\cline { 1 - 5 } & P1 & P2 & P3 & P4 & P5 & P6 & P7 & P8 & P9 \\
1. Elementos da & 1 & 1 & 1 & 1 & 1 & 1 & 1 & 0 & 1 \\
mão humana & 0 & 0 & 0 & 0 & 0 & 0 & 1 & 0 & 1 \\
incorporados na & 0 & 0 & 0 & 0 & 0 & 0 & 0 & 0 & 1 \\
prótese: & 1 & 1 & 1 & 1 & 1 & 0 & 1 & 0 & 1 \\
& 0 & 0 & 0 & 0 & 0 & 0 & 0 & 0 & 0
\end{tabular}

\begin{tabular}{lccccccccc} 
2. Elementos & 0 & 1 & 1 & 0 & 0 & 0 & 1 & 0 & 1 \\
funcionais expostos & 1 & 1 & 0 & 1 & 1 & 1 & 1 & 0 & 1 \\
na prótese: & 1 & 1 & 0 & 1 & 1 & 1 & 1 & 1 & 1 \\
& 0 & 1 & 0 & 0 & 0 & 0 & 0 & 0 & 1 \\
& 0 & 0 & 1 & 0 & 0 & 0 & 0 & 0 & 0 \\
\hline & 4 & $\mathbf{6}$ & $\mathbf{4}$ & $\mathbf{4}$ & $\mathbf{4}$ & $\mathbf{3}$ & $\mathbf{6}$ & $\mathbf{1}$ & $\mathbf{8}$
\end{tabular}

\footnotetext{
*As próteses analisadas foram nomeadas na tabela como P1= Raptor Reloaded, $\mathrm{P} 2=$ Phoenix Hand, P3 = Osprey Hand, P4 = Cyborg Beast, P5 = Raptor Hand, P6 = Taylor hand 2x,P7= K-1 Hand, P8 = Odysseus Hand, P9 = Flexy Hand 2
} 
Tabela 3 - Tabela de similaridade antropomórfica. fonte. Imagens das Próteses analisadas 1: Raptor Reloaded. 2: Phoenix Hand. 3: Osprey Hand. 4: Raptor. 5: Talon Hand. 6: Cyborg Beast. 7: Odysseus Hand. 8: Flexy Hand 2. 9: K1 Hand. Imagens adaptadas de: 1:(E-nabling The future, 2016d); 2: (E-nabling The future 2016e); 3:(E-nabling The future, 2016f); 4:(E-nabling The future, 2016g); 5: (E-nabling The future, 2016h); 6: (E-nabling The future, 2016i); 7: (E-nabling The future, 2016j) 8: (E-nabling The future, 2016k); 9: (E-nabling The future, 2016l).

\section{FABRICAÇÃO E MANUTENÇÃO}

Todos os projetos são desenvolvidos visando a fabricação de suas partes por meio da impressão 3D, contudo, necessitam, obrigatoriamente, de outros componentes comercialmente disponíveis para a montagem da prótese. Todos os dispositivos preveem o uso de fios tensionadores para ativação do movimento de abrir e fechar dos dedos do elemento terminal das próteses. $\mathrm{O}$ uso de parafusos e/ou rebites para a união das partes e o ajuste do tensionamento dos fios, velcro para a fixação e ajuste da prótese no antebraço do usuário e material acolchoado/couro para o interior do soquete da prótese, que devem ser adquiridas comercialmente. A manutenção das próteses é realizada através da reimpressão das partes danificadas ou da recompra dos componentes comerciais.

\section{INSTRUÇÕES E TREINAMENTO E SEGURANÇA}

Todas as próteses são montadas a partir das orientações fornecidas por seus respectivos manuais de instruções, que são disponibilizados nas páginas de download das próteses, nos sites http://thingverse.com e http://3dprint.nih.gov/ (K1 Hand). Os manuais expõem as etapas de montagem das próteses e orientam acerca dos materiais que devem ser adquiridos comercialmente, bem como, da quantidade dos mesmos. Além disso, vídeos com tutoriais das montagens ou partes das montagens podem ser encontrados nas páginas de download de suas respectivas próteses.

Todos os manuais são escritos apenas em língua inglesa, com exceção do manual da Raptor Hand, que representa a montagem utilizando apenas imagens, restringindo a parte escrita às especificações dos materiais. Por sua vez, para a montagem da Raptor Reloaded, há a possibilidade de acessar o site http://www. handchallenge.com/, que disponibiliza vídeos que auxiliam no processo de montagem da prótese, voltados para crianças. No que tange a aquisição dos dados antropométricos para a adaptação dimensional da prótese, apenas os manuais da Cybor Beast e Osprey Hand, (CYBORGBEAST, 2016b);(Osprey Hand Assembly Instructions, 2016) abordam o assunto.

Não são apresentadas instruções de segurança específica para cada dispositivo nos respectivos manuais de instruções, apenas a Odysseus Hand, cita na página de 
download dos arquivos, que a prótese possui dedos mais largos, que diminuem a possibilidade do usuário criança ferir-se devido a agudeza dos dedos (THINGIVERSE, 2016h).

O usuário consegue obter informações de segurança tocante a todos os dispositivos, no site da E-nabling the Future, no link "Safety Guide Lines" (http://enablingthefuture.org/safety-guidelines/). O safety guide lines esclarece ao usuário que as próteses são consideradas dispositivos experimentais que não são capazes de segurar objetos pesados e não substituem o membro amputado do usuário. Além de oferecer dicas de segurança para os seguintes tópicos: experimentação com impressoras 3D, segurança da saúde, segurança da criança e instruções de limpeza.

\section{CONFORTO}

Considerando o delineamento do presente estudo, não há como analisar a percepção de conforto dos usuários quanto ao uso das próteses. Por tratar-se de um aspecto subjetivo, este estudo deveria ser realizado mediante a coleta de informações com usuários específicos e com metodologia adequada. Todavia, observa-se que as próteses analisadas possibilitam ajustes em suas interfaces, que podem influenciar diretamente no conforto percebido pelos usuários. Esse ajuste é relativo às dimensões das partes da prótese, pois os arquivos digitais oferecem a possibilidade de redimensionamento em software CAD, permitindo que a mesma se ajuste à antropometria do indivíduo. Um ajuste fundamental é o do soquete em relação às dimensões do pulso do usuário. Para auxiliar o processo de adaptação dimensional da prótese, o site da E-nabling the Future disponibiliza o software Handomatic, que permite que o usuário modifique os arquivos STL com dimensões personalizadas às medidas e deformidades do usuário.

Para partes que entram em contato direto com a pele do usuário, como a região palmar da prótese que abriga o coto, os desenvolvedores sugerem a inserção de uma manta acolchoada no interior da região, visando diminuir o desconforto. Outros elementos importantes para a adaptação da prótese ao usuário são as faixas de velcro que envolvem o braço do paciente, que oferecem livre ajuste para a fixação da prótese.

\section{ASPECTOS FUNCIONAIS}

Todas as próteses são mecânicas, ativadas com a energia do corpo, mais especificamente pela articulação do pulso, sendo necessário ao usuário possuir nível de amputação parcial da mão, carpal ou transcarpal, que preservam a articulação do pulso. Essa articulação será a responsável pela execução do movimento de flexão, que permite o fechamento dos dedos da prótese, e o de extensão, que permite a abertura 
dos dedos da prótese. Os cinco dedos são ativados concomitantemente, a partir destes movimentos. A exceção é a Odysseus Hand que possui apenas 03 dedos, porém são acionados concomitantemente, como os demais.

Duas próteses apresentam alternativas para reduzir a força de flexão necessária à abertura dos dedos da prótese. A Flexy Hand 2 possui articulações dos dedos em material flexível (PLA flexível), para que os mesmos retornem automaticamente à posição aberta (THINGIVERSE, 2016c). O mesmo princípio de retorno é adotado na Phoenix Hand v2, por meio de elásticos ortodônticos acoplados na parte superior das articulações interfalângicas proximais da prótese (DIAMOND, 2016). A Odysseus Hand possui apenas três dedos largos, com o propósito de diminuição da força aplicada por crianças durante o uso da prótese, uma vez que a ela enfrenta menor resistência ao realizar o movimento de flexão durante a utilização da mesma.

\section{DISCUSSÃO}

A análise das características projetuais e de distribuição das próteses organizadas pela iniciativa E-nabling the Future evidenciam a ênfase da comunidade em desenvolver e disponibilizar gratuitamente próteses funcionais com custo de fabricação e manutenção reduzidos. São condizentes, portanto, com os aspectos básicos do open source: a livre distribuição, uso e modificação das próteses. Os desenvolvedores asseguram o acesso aos dispositivos em diferentes partes do planeta, ao disponibilizar os arquivos para a sua produção em plataformas online. Ainda, a produção por meio da impressão 3D, além de reduzir o tempo de produção das próteses, garante maior durabilidade aos dispositivos, visto que as partes das próteses podem ser reimpressas tão logo forem danificadas. Não menos importante, a utilização de componentes de montagem simples como parafusos e fios tensionadores podem ser, facilmente, adquiridos em mercados locais e auxiliam na redução de custos e na manutenção. E esse último, somado à durabilidade, configuram aspectos fundamentais para dispositivos utilizados por crianças em estágios de crescimento.

A utilização da impressão 3D e a maneira com que os elementos das próteses (soquete e dispositivo terminal) da E-Nabling the Future configuram-se e unem-se, conferem modularidade aos dispositivos, facilitando a personalização de cada um dos elementos da prótese, através de adaptações dos elementos ou o desenvolvimento de novas soluções para os mesmos. Em razão dessa característica, as próteses estão em constante aprimoramento, graças ao estabelecimento de uma comunidade sólida de desenvolvedores, que elaboram experimentações em elementos dos projetos que podem ser implementados em versões futuras dos dispositivos ou em personalizações implementadas pelo próprio usuário. 
Exemplos dessa experimentação podem ser observados nos fóruns online do site da E-Nabling The Future, que apresenta propostas de melhorias mecânicas nos dedos das próteses (PHPBB, 2015a), no aprimoramento do processo de impressão e montagem das partes (PHPBB, 2015b), avanços de sistemas de fixação no membro do indivíduo (PHPBB, 2015c) e estética das próteses (PHPBB, 2015d).

Porém, essas experimentações carecem de validação consistente no que tange aos aspectos da usabilidade, pois os projetos desenvolvidos pela comunidade são testados, em grande parte, pelos próprios desenvolvedores que também podem ser os usuários finais. Essa limitação também é observada em versões oficiais das próteses, como pode ser verificado na descrição do desenvolvedor sobre a Talon Hand 3.0 (THINGIVERSE, 2016e), na qual o desenvolvedor afirma ter empregado melhorias no acolchoamento interno da prótese, corroborado pelo feedback de seu filho. Essa prática de projeto vai contra o que Martin et al (2010) sugerem para validar conceitos com usuários, que segundo os autores, devem ser realizados por meio de técnicas de inferência (entrevistas, focous groups, questionários) em grupos de usuários, dos modelos mentais do usuário acerca do funcionamento do produto, dos usos inadequados, dos erros potenciais e opinião geral sobre o design do dispositivo. Portanto, observa-se nos dispositivos analisados que o levantamento de requisitos do projeto está atrelado ao fato dos desenvolvedores serem, muitas vezes, indivíduos com amputação.

No que tange aos aspectos relativos à segurança, observa-se a falta de informações nos manuais de instrução relativos à utilização adequada e as limitações funcionais das próteses. Já no que concerne ao aspecto estético dos dispositivos, percebe-se uma tendência da estética limitada aos aspectos funcionais dos dispositivos, ou seja, as soluções formais privilegiam a montagem, a segurança e a execução das funções das próteses. Porém, o aspecto cosmético relacionado à similaridade do membro colateral ainda é pouco observado. Vasluian et al. (2013) apresenta este como sendo o aspecto mais importante para a aceitação da prótese pelo indivíduo. Mas apenas no projeto da Flexy Hand 2 parece ter sido desenvolvido o aspecto estético com o objetivo de assemelhar-se à mão humana.

Já no que diz respeito ao acesso dos usuários as próteses, verifica-se uma eventual limitação relacionada a questões linguísticas, visto que, os sites para o download dos dispositivos, estão em língua inglesa. O mesmo ocorre como toda a documentação referente à montagem das próteses. Isso exige que o usuário tenha conhecimento sobre a língua inglesa para uma ideal interpretação e assimilação dos conteúdos apresentados.

Por compor o rol dos dispositivos open hardware, a fabricação das próteses disponibilizadas pela E-Nabling the Future está atrelada à necessidade do usuário em 
adquirir os componentes comerciais da prótese, que vão desde os filamentos plásticos para a impressão 3D, até parafusos para montagem. A aquisição desses componentes pode ser restringida por aspectos legais. Por exemplo, na Argentina as leis nacionais impedem que pessoas físicas importem determinados materiais que compõem as próteses (E-NABLING THE FUTURE, 2016m). Ainda, para efetiva adaptação de suas partes, adequação antropométrica e impressão das próteses, é necessário que os usuários/ fabricantes possuírem competência na manipulação de softwares de modelagem 3D e na operação de impressoras 3D. Esse fato dificulta a inserção das próteses open source em países em desenvolvimento e zonas de guerra, nas quais a população carece desses dispositivos. Segundo Ishengoma; Mtaho (2014) essas regiões são desprovidas de um número consistente de técnicos especializados na fabricação de próteses, que por vezes exige que o desenvolvimento em software CAD seja realizado em outro país, a partir da aquisição dos dados antropométricos do paciente realizado em seu país. Ademais essas regiões possuem acesso restrito a impressoras 3D, cuja presença dos referidos equipamentos pode estar limitada a maker spaces, como os FabLabs.

Ainda no que tange à limitação ao acesso às próteses open source, evidencia-se nessas regiões do planeta uma clara limitação quanto à disponibilidade de internet, requisito básico para a obtenção dos arquivos das próteses. Os índices de acesso à mesma, em países em desenvolvimento são baixos, como por exemplo, na Índia (20\% da população), Bangladesh ( $11 \%$ da população) e Paquistão ( $8 \%$ da população), de acordo com PewResearch (2015). Em alguns países africanos as condições são mais precárias ainda, considerando a baixíssima penetração da internet, como nos casos da Etiópia, Guinea e Nigéria, que apresentam índices de acesso abaixo dos $2 \%$ em relação a suas populações (NYIRENDA-JERE; BIRU, 2015).

É indispensável ressaltar o fato das próteses disponibilizadas no site E-Nabling the Future serem consideradas pelos próprios autores dispositivos experimentais, que carecem de testes biomecânicos consistentes em situações reais de uso. Considerando esse aspecto, Dally et al (2015) realizaram testes de AVD com 40 indivíduos sem amputação, usando a prótese Raptor Reloaded, no qual notou-se a impossibilidade da prótese em executar ações que requerem precisão. Ainda, observou-se que versões em escalas reduzidas do dispositivo tiveram dificuldades para erguer pequenas cargas. Isso pode estar relacionado com a posição não natural do polegar da prótese, pois este elemento localiza-se na lateral das próteses e é incapaz de realizar o movimento de abdução no plano da mão, bem como, o movimento de oposição. Contudo, o autor, sinaliza o uso satisfatório para crianças nas AVD como folhear livros, devido às pequenas cargas que estas atividades exigem.

Diante do exposto, sugerem-se para o aprimoramento dos projetos de próteses open source, relacionadas aos fatores humanos, os seguintes fatores: I) O desenvolvimen- 
to de protocolos padronizados para a validação dos aspectos funcionais, ergonômicos e de satisfação do usuário e sua disponibilização aos desenvolvedores/usuários, evitando a pura experimentação; II) O desenvolvimento de manuais de instruções mais intuitivos, reduzindo a necessidade de textos, evitando dificuldades na interpretação de outros idiomas; III) Inclusão de mais opções de idiomas no conteúdo escrito dos sites nos quais os arquivos estão disponibilizados; IV) Explicitação dos dados de segurança e uso adequado das próteses nos manuais de instruções; V) Desenvolvimento de próteses com maior antropomorfismo, visando o engajamento do usuário.

\section{CONCLUSÃO}

As próteses open source, voltadas para a fabricação em impressão 3D, levam em consideração os aspectos de interação física, funcional e financeira de indivíduos amputados, sobretudo, as questões pertinentes ao contexto social e a adaptação da prótese à antropometria do membro, ou seja, os fatores humanos e ergonômicos dos dispositivos.

Apesar da consistência dos projetos aos princípios do open design (que engloba o open source e o open hardware), as próteses open source podem enfrentar desafios na sua distribuição efetiva em regiões pouco desenvolvidas do planeta, devido à baixa infraestrutura física desses locais, à limitação no acesso à internet e precariedade na disponibilidade de software CAD e impressoras 3D, aspectos indispensáveis para a construção, montagem e manutenção das próteses.

Os aspectos analisados e discutidos que dizem respeito aos fatores humanos e ergonomia, levam ao questionamento da consistência desses aspectos, umas vez que, a implementação dos mesmos, com frequência, se efetiva em decorrência da experimentação de usuários, sem avaliações formais. No entanto, é perceptível que próteses open source apresentam um ganho na possibilidade da acessibilidade do ponto de vista financeiro, tanto no âmbito da fabricação quanto da manutenção. Assim, próteses open source fabricadas em impressão 3D tem o potencial para atuar como alternativas viáveis às próteses tradicionais, contribuindo com a melhoria na realização de atividades pontuais, como lazer e estudo, possibilitando a opção de tratamento prostético a indivíduos que, possivelmente, não teriam acesso às mesmas por meios tradicionais.

\section{REFERÊNCIAS}

ACOSTA, Roberto et al. Open source hardware. 2009. Tese de Doutorado. Massachusetts Institute of Technology.

ANDERSON, Chris. Makers. Nieuw Amsterdam, 2013. 
AVITAL, M. The Generative Bedrock Of Open Design. In: VAN ABEL, Bas et al. Open design now: why design cannot remain exclusive. BIS Publishers, 2011. Disponível em http://opendesignnow.org/index.html\%3Fp=405.html Acesso em Agosto de 2016.

BERMAN, Barry. 3-D printing: The new industrial revolution. Business horizons, v. 55, n. 2, p. 155-162, 2012.

BURN, Matthew B.; TA, Anderson; GOGOLA, Gloria R. Three-Dimensional Printing of Prosthetic Hands for Children. The Journal of hand surgery, 41(5). 2016. p103-p109. Diponível em: http://www.sciencedirect.com/science/article/pii/S0363502316002136. Acesso em Agosto de 2016.

CLEMENT, R. G. E.; BUGLER, K. E;; OLIVER, C. W. Bionic prosthetic hands: A review of present technology and future aspirations. The surgeon, v. 9, n. 6, p. 336-340, 2011. Diponível em: http:// www.sciencedirect.com/science/article/pii/S1479666X11000904 Acesso em Agosto de 2016.

CUMMINGS, D. Prosthetics in the developing world: a review of the literature.Prosthetics and orthotics international. Glasgow, v.20.1 1996. Disponível em: http://www. oandplibrary.org/poi/1996 01 051.asp Acesso em Agosto 2016.

COOK, Albert M.; POLGAR, Janice Miller. Assistive technologies: Principles and practice. Elsevier Health Sciences, 2014.

CONNER, Brett P. et al. Making sense of 3-D printing: Creating a map of additive manufacturing products and services. Additive Manufacturing, v. 1, p. 64-76, 2014.

CORDELLA, Francesca et al. Literature review on needs of upper limb prosthesis users. Frontiers in neuroscience, v. 10, 2016.

CYBORGBEAST. About Us. Disponível em: http://www.cyborgbeast.org/\#/about. Acesso em Agosto 2016a.

CYBORGBEAST. Tutorials. Disponível em: http://www.cyborgbeast.org/\#/tutorials. Acesso em 2016b.

DALLY, Corinne, et al. Characteristics of a 3D-printed prosthetic hand for use in developing countries. Global Humanitarian Technology Conference (GHTC), 2015 IEEE. IEEE, 
2015. Disponível em: http://ieeexplore.ieee.org/xpls/abs all.jsp?arnumber $=7343956$ Acesso em Agosto de 2016

DAVIDSON, Judith. A survey of the satisfaction of upper limb amputees with their prostheses, their lifestyles, and their abilities. Journal of Hand Therapy, v. 15, n. 1, p. 62-70, 2002.

DE MUL, Jos. Redesigning design. Open Design Now: Why Design Cannot Remain Exclusive. Amsterdam: BIS publishers, 2011.

DIAMOND, Jonh. Assembvling the Phoenix hand, 2016. Disponível em: https://thingiverse-production-new.s3.amazonaws.com/assets/dd/6b/45/30/fc/Phoenix v2 assembly guide.pdf. Acesso em Agosto de 2016.

ELMANSY, Rafik. Designing the 3D-Printed Prosthetic Hand. Design Management Review, 2015, v.26n.1 p. 24-31.

E-NABLING THE FUTURE. About Us. Disponível em: http://enablingthefuture.org/ about/. Acesso em agosto de 2016a.

E-NABLING THE FUTURE. FAQS(General). Disponível em: http://enablingthefuture. org/fags-general/. Acesso em agosto de 2016b.

E-NABLING THE FUTURE. Media FAQ. Disponível em: http://enablingthefuture.org/ faqs/media-faq/. Acesso em agosto de 2016c.

E-NABLING THE FUTURE. The raptor Reloaded. Disponível em: http://enablingthefuture.org/upper-limb-prosthetics/raptor-reloaded/ Acesso em Agosto 2016d.

E-NABLING THE FUTURE. Phoenix Hand. Disponível em: http://enablingthefuture.org/ phoenix-hand/. Acesso em Agosto de2016e.

E-NABLING THE FUTURE. Osprey Hand. Disponível em: http://enablingthefuture.org/ osprey-hand/. Acesso em Agosto de $2016 f$.

E-NABLING THE FUTURE. The Raptor Hand. Disponível: http://enablingthefuture.org/ upper-limb-prosthetics/the-raptor-hand/. Acesso em $2016 \mathrm{~g}$. 
E-NABLING THE FUTURE. Talon Hand 2.x. Disponível em: $\underline{\text { http://enablingthefuture.org/ }}$ upper-limb-prosthetics/talon-hand/. Acesso em 2016h.

E-NABLING THE FUTURE. The Cyborg Beast. Disponível em: http://enablingthefuture. org/upper-limb-prosthetics/cyborg-beast/. Acesso em $2016 \mathrm{i}$.

E-NABLING THE FUTURE. The Odysseus Hand. Disponível em: http://enablingthefuture.org/upper-limb-prosthetics/odysseus-hand/. Acesso em 2016j.

E-NABLING THE FUTURE. The Flexy Hand And Flexy Hand 2. Disponível em: http://enablingthefuture.org/upper-limb-prosthetics/the-flexy-hand/. Acesso em Agosto 2016k.

E-NABLING THE FUTURE. K1 Hand. Disponível em: http://enablingthefuture.org/k-1hand/. Acesso em Agosto 2016l.

E-NABLING THE FUTURE .Making Hands In Argentina. Disponível em: http://enablingthefuture.org/2014/05/01/making-hands-in-argentina/. Acesso em Agosto 2016m.

GILJA, Vikash et al. Clinical translation of a high-performance neural prosthesis. Nature medicine,Nat Med. ; 21(10): 1142-1145 Out. 2015. Disponível em: http://europepmc. org/articles/pmc4805425 Acesso em Agosto de 2016.

HECKATHORNE, Craig W. Upper-Limb Prosthetics. In: OLSON, Don A.; DERUYTER, Frank. Clinician's guide to assistive technology. St. Louis, Mo: Mosby, 2002. Cap. 16. p. 265-277.

INSTRUCTABLES. Assembly of e-NABLES's Raptor Reloaded Hand. Disponível em: http://www.instructables.com/id/Assembly-of-e-NABLEs-Raptor-Reloaded-Hand/. Acesso em Agosto de 2016.

ISHENGOMA, Fredrick R.; MTAHO, Adam B. 3D printing: developing countries perspectives. International Journal of Computer Applications v104-n11, 2014. Disponível em: http://arxiv.org/abs/1410.5349. Acesso em Agosto 2016.

KADUSHIN, Ronen. Open design manifesto. Ronan Kadushin Design, 2010. Disponível em: http://www.ronen-kadushin.com/files/4613/4530/1263/Open Design Manifesto-Ronen Kadushin .pdf. Acesso em Novembro 2016. 
KATZ, A. Authors and Owners. In: VAN ABEL, Bas et al. Open design now: why design cannot remain exclusive. BIS Publishers, 2011. Disponível em : http://opendesignnow. org/index.html\%3Fp=407.html Acesso em Agosto de 2016.

LANG, Alexandra R. et al. The effect of design on the usability and real world effectiveness of medical devices: a case study with adolescent users. Applied ergonomics, v. 44 , n. 5, p. 799-810, 2013

LIPSON, Hod; KURMAN, Melba. Fabricated:The new world of 3D printing. John Wiley \& Sons, 2013.

MARTIN, J. L. et al. Design for patient safety: User testing in the development of medical devices. London: National Patient Safety Agency, 2010.

MINISTÉRIO DA SAÚDE. Diretrizes de Atenção à Pessoa Amputada. Secretaria de Atenção à Saúde Departamento de Ações Programáticas Estratégicas, 2a edição, 2014.

MUSICUS, M; DAVIS, A. Upper extremity prosthetic design and function. In: SPIRES, Mary Catherine; KELLY, Brian M.; DAVIS, Alicia J. Prosthetic Restoration and Rehabilitation of the Upper and Lower Extremity. Demos Medical Publishing, 2013. Cap. 15. p. 167-178

NGD - National Guideline Clearinghouse. VA/DoD Evidence-Based Clinical Practice Guideline for the Management of Upper Extremity Amputation Rehabilitation. V1.0, 2014. Disponível em: https://www.guideline.gov/summaries/summary/48529/ vadod-clinical-practice-guideline-for-the-management-of-upper-extremity-amputation-rehabilitation. Acesso em agosto de 2016

NEVES, Heloisa Maria Domingues. Maker innovation. Do open design e fab labs... às estratégias inspiradas no movimento maker. Tese de Doutorado. Universidade de São Paulo, 2014

NIH. ThE k1 Hand by Evan Keuster. Disponível em: http://3dprint.nih.gov/discover/3DPX-001705.

NYIRENDA-JERE, Towela; BIRU, Tesfaye. Internet development and Internet governance in Africa. Internet Society, Geneva, 2015. Disponível em: http://www.internetsociety. 
org/sites/default/files/Internet\%20development\%20and\%20Internet\%20governance\%20in\%20Africa.pdf. Acesso em Agosto de 2016.

Osprey Hand Assembly Instructions. Disponpivel em: https://docs.google.com/ document/d/1xbfhPYKO-4hcpnAmYpvGPY-vzPpO10TiwvFZkcN3034/edit. Acesso em Agosto de 2016.

PEW RESEARCH CENTER. Internet Seen as Positive Influence on Education but Negative on Morality in Emerging and Developing Nations. 2015. Disponível em: http://www.pewglobal.org/2015/03/19/internet-seen-as-positive-influence-on-education-but-negative-influence-on-morality-in-emerging-and-developing-nations/. Acesso em Agosto de 2016.

PHPBB. Rubber Band Raptor Reloaded. E-NABLE Forums. 2015a. Disponível em: http:// forums.e-nable.me/viewtopic. php?f=9\&t=135. Acesso em Agosto de 2016.

PHPBB. New Print-in-Place Fingers: Three Phalanges, No Elastics. E-NABLE Forums. 2015b. Disponível em: http://forums.e-nable.me/viewtopic.php?f=9\&t=221. Acesso em Agosto 2016.

PHPBB. Replacing the Velcro on the Gauntlet: Two Printable Solutions. E-NABLE Forums. 2015c. Disponível em: http://forums.e-nable.me/viewtopic. php?f=9\&t=161. Acesso em Agosto 2016.

PHPBB. Handaptive: a new approach for parametric hand design. E-NABLE Forums. 2015d. Disponível em: http://forums.e-nable.me/viewtopic.php?f=9\&t=161. Acesso em Agosto 2016.

RESNIK, Linda, et al. Advanced upper limb prosthetic devices: implications for upper limb prosthetic rehabilitation. Archives of physical medicine and rehabilitation 93.4 2012. p.710-717. Disponível em: http://www.ncbi.nlm.nih.gov/pubmed/22464092 Acesso em Agosto de 2016.

ROBOHAND. Press Package: Disponível em: http://www.robohand.net/press-package/ . Acesso em Agosto 2016a.

ROBOHAND. Faq. Disponível em: http://www.robohand.net/faq/. Acesso em agosto 2016b.

116 
SLADE, Patrick, et al. Tact: Design and performance of an open-source, affordable, myoelectric prosthetic hand 2015 IEEE International Conference on Robotics and Automation (ICRA). IEEE, 2015. Disponível em http://ieeexplore.ieee.org/xpl/login. jsp?tp=\&arnumber $=7140105 \&$ url=http $\% 3$ A\%2F\%2Fieeexplore.ieee.org\%2Fiel7\%2F7128761\%2F7138973\%2F07140105.pdf\%3Farnumber\%3D7140105 Acesso em Agosto de 2016.

STRAIT, Erin. Prosthetics in developing countries. Prosthetic Resident, 2006. Disponível em: http://www.oandp.org/publications/resident/pdf/DevelopingCountries.pdf. Acesso em Agosto 2016

SHARPLES, Sarah et al. Medical device design in context: A model of user-device interaction and consequences. Displays, v. 33, n. 4, p. 221-232, 2012.

THINGIVERSE. Cyborg Beast. Disponível em: http://www.thingiverse.com/ thing:261462. Acesso em Agosto 2016a.

THINGIVERSE. E-NABLE Phoenix hand v2. Disponível em: http://www.thingiverse. com/thing:1453190. Acesso em Agosto 2016b.

THINGIVERSE. Flexy-Hand 2. Disponível em: http://www.thingiverse.com/ thing:380665. Acesso em Agosto 2016c.

THINGIVERSE. Raptor Reloaded by e-NABLE. Dispnível em: http://www.thingiverse. com/thing:596966. Acesso em Agosto 2016d.

THINGIVERSE. Talon Hand 3.0. Disponível em: http://www.thingiverse.com/ thing:229620. Acesso em Agosto de 2016e.

THINGIVERSE. The Osprey Hand by Alderhand and e-nable. Disponível em: https:// www.thingiverse.com/thing:910465. Acesso em Agosto 2016f.

THINGIVERSE. The Raptor Hand by e-NABLE. Disponível em: http://www.thingiverse. com/thing:476403. Acesso em Agosto 2016g.

THINGIVERSE. Ody Hand 2.0. Disponivel em: http://www.thingiverse.com/thing:262930. Acesso em Agosto 2016h. 
VALPREDA, F. 3D printing awareness: the future of making things. In:IS\&T/SPIE Electronic Imaging. International Society for Optics and Photonics, 2015.

VASLUIAN, Ecaterina et al. Opinions of youngsters with congenital below-elbow deficiency, and those of their parents and professionals concerning prosthetic use and rehabilitation treatment. PloS one, v. 8, n. 6, p. e67101, 2013.

WORLD HEALTH ORGANIZATION et al. Medical device regulations: Global overview and guiding principles. 2003.

World Health Organization. World report on disability. World Health Organization, 2011. Diponível em: http://www.who.int/disabilities/world report/2011/en/ Acesso em Agosto de 2016.

YANAGISAWA, Takufumi et al. Electrocorticographic Control of a Prosthetic Hand in Paralyzed Patients. In: Brain-Computer Interface Research. Springer Berlin Heidelberg, 2014. p. 95-103. Diponível em: http://onlinelibrary.wiley.com/doi/10.1002/ ana.22613/abstract Acesso em Dezembro de 2015.

ZLOTOLOW, Dan A.; KOZIN, Scott H. Advances in upper extremity prosthetics. Hand clinics, v. 28, n. 4, p. 587-593, 2012. Diponível em: http://www.sciencedirect.com/science/ article/pii/S0749071212001102 Acesso em Dezembro de 2015.

ZUNIGA, Jorge, et al. Cyborg beast: a low-cost 3d-printed prosthetic hand for children with upper-limb differences. BMC research notes, v.8n.1. 2015. Disponível em: $h$ ttps:// www.ncbi.nlm.nih.gov/pmc/articles/PMC4304188/. Acesso em Agosto 2016. 
Ricardo Schwinn Rodrigues possui graduação em Design Industrial pela Universidade do Estado de Santa Catarina-UDESC Tem experiência na área de Desenho Industrial, com ênfase em Desenho de Produto. Mestrando no Programa de Pós-Graduação em Design da Universidade do Estado de Santa Catarina-UDESC.

Susana Cristina Domenech possui graduação em Licenciatura Plena em Química e em Química pela Pontifícia Universidade Católica do Rio Grande do Sul (1992), Mestrado em Engenharia de Minas, Metalúrgica e de Materiais pela Universidade Federal do Rio Grande do Sul (1997) e Doutorado em Engenharia de Materiais pela Universidade Federal de Santa Catarina (2002) - Doutorado sanduíche (CNPq) pela Martin-Luther Universität Halle-Wittenberg (Alemanha) e Albert-Ludwigs Universität Freiburg (Alemanha). Atualmente é Professor Associado da Universidade do Estado de Santa Catarina no Centro de Ciências da Saúde e do Esporte, Professor permanente no Programa de Pós-Graduação em Ciências do Movimento Humano da UDESC e no Programa de Pós-Graduação em Design da UDESC.

Milton José Cinelli possui graduação em Física Bacharelado pela Universidade Federal de Santa Catarina (1989), mestrado em Física pela Universidade Federal de Santa Catarina (1992) e doutorado em Chimie Physique Option Matériaux - Université de Limoges - França (1996). Professor adjunto e Professor Permanente do Programa de Pós-Graduação em Design na linha Interfaces e Interações Físicas da Universidade do Estado de Santa Catarina. Tem experiência na área de Física, atuando principalmente nos seguintes temas: Tecnologias Assistivas, Metrologia, Técnicas Gerais de Laboratório, Sistema de Instrumentação. Atualmente é Coordenador de Pesquisa Institucional.

Marcelo Gitirana Gomes Ferreira is an associate professor at the State University of Santa Catarina (UDESC - Brazil). He received his Dr. Eng. degree in Mechanical Engineering at the Federal University of Santa Catarina (UFSC) in 2006. From 1998 to 2002, he worked as product engineer for Volkswagen in Brazil and also in Germany (Wolfsburg). His research interests include Project Management, Product Development Process, and Human Factors.

Recebido em: 16/08/2016

Aceito em: 22/11/2016 